\title{
Data Privacy and Security Considerations for Personal Assistants for Learning (PAL)
}

Elaine M. Raybourn ${ }^{\text {ab }}$, Nathan Fabiana, Warren L. Davis IVa, Ray Parks ${ }^{a}$, Jonathan T. McClain ${ }^{a}$, Derek Trumbo ${ }^{a}$, Damon Regan ${ }^{b}$, Paula J. Durlach ${ }^{b}$

\section{INTRODUCTION}

A future PAL, not unlike current iterations of virtual assistants, would make recommendations and monitor user activities in realtime. It has the ability to infer the user's needs and respond by making recommendations for learning. One approach to achieving this vision is to foster interoperability among existing and future heterogeneous systems, all of which can contribute some knowledge of the user to a persistent user model [1, 2]. There are many social and technical challenges associated with privacy control and protection, or security, of personal data. For the PAL vision to succeed, users will need to feel that their data are safe with a PAL. They will also need to trust the PAL to use their information so that it meets their evolving requirements for privacy.

This poster identifies some typical risks associated with user models and intelligent systems, and proposes mitigation strategies. In truth, it is not really possible to secure a system perfectly and indefinitely against motivated attacks. The questions instead are "how long is it protected?" and "how much is protected?" The answers will not be "forever" and "all." Instead data privacy concerns and security strategies for PAL must be considered an ongoing effort. The risks and proposed mitigations below are areas of research under consideration for PAL.

\section{in}

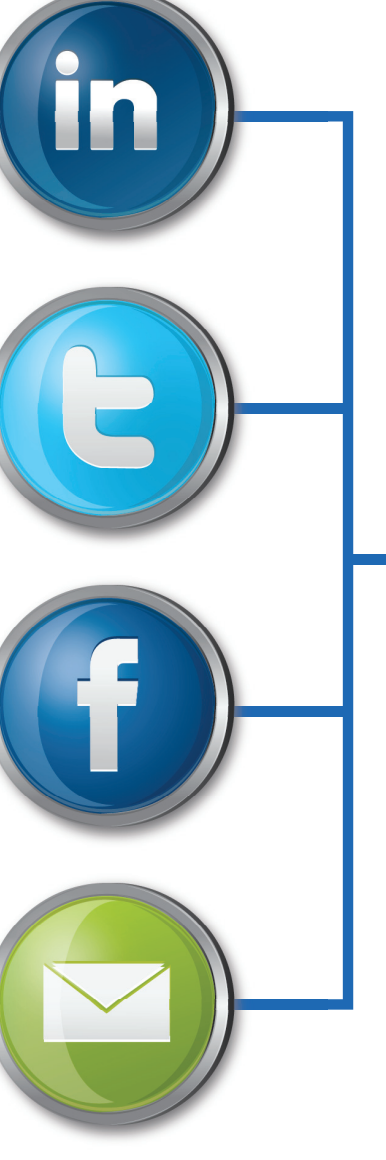

\begin{tabular}{|r|l|}
\hline \multicolumn{2}{|c|}{ User Model Components } \\
\hline Components & Description \\
\hline Profile & $\begin{array}{l}\text { Basic information of the user (e.g., user identification, favorite subjects, } \\
\text { hobbies, aspirations, goals) }\end{array}$ \\
\hline Education \& Training & Education and training history, grades, and feedback \\
\hline Career & User's activities that demonstrate capabilities \\
\hline Qualification & $\begin{array}{l}\text { Official evidence data (e.g., academic transcripts, professional/vocational } \\
\text { qualifications, certificates, licenses, and letters of recommendation) }\end{array}$ \\
\hline Experience & Extra-curricular activities (e.g., clubs, internships, volunteer activities) \\
\hline Outcome & $\begin{array}{l}\text { Digital and non-digital artifacts that resulted from learning experience } \\
\text { (e.g., documents, photos, animations, videos, audio files, images) }\end{array}$ \\
\hline Feedback & Feedback from instructors, peers, and others from the learning process \\
\hline Reflection & $\begin{array}{l}\text { Personal descriptions (e.g., comments, explanations, etc.) about learning } \\
\text { or teaching activities including perceived strengths and weaknesses }\end{array}$ \\
\hline
\end{tabular}

\section{USER MODELS}

Open learner models are defined as user models that are accessible to the user being modeled or possibly to teachers, peers, or others who may be able to enhance the model [3]. In addition to improved accuracy, open learner models are thought to enhance metacognition, motivation, and collaboration and/or competition. Profile, career, education, awards, and training history, and qualifications may be captured from sites such as Linkedln, etc. Daily experience, links, feedback from peers or instructors, and sources of reflection could be captured from sites such as Facebook and Twitter.

Risk: A user may falsify one's own performance data (e.g. test scores, certifications, grades, etc.) to manipulate course outcomes.

\section{REFERENCES}

Kay, J. and Kummerfeld, B. Lifelong learner modeling. In P. Durlach \& A Lesgold (Eds.), Adaptive Technologies for Training and Education. Cambridge Kuffik, T, Press (2012), 140-164

user modeling In A. Krummer \& T. Ksvi (Eallenges and solutions of ubiquitous Ker \&. Ksvi (Eds.), Ubiquitous Display Environments. Spetrieved January 14, 2015 from: http://sydney.edu. au/engineering/it/ judy/Homec/Pubs/2012_Ubiquitous___Ser_Modeling.pdf. Bull, S. and Kay, J. Student models that invite the learner in: The SMLI:O) ope learner modelling framework. Internation
in Education (2007), 17(2), 89-120.

Compañó, R. and Lusoli, W. The policy maker's anguish: Regulating personal data behavior between paradoxes and dilemmas. Eighth Workshop on the Economics of Information Security, London, UK (2009), 1-16.
Mitigation: Manipulations of this kind would present as statistical anomalies in the data and could therefore be detected through common analysis.

Risk: PAL devices may have access to sensors, socia media sites, or multiple apps installed (not all of which are dedicated to PAL).

Mitigation: Understand how data can be harvested from sensors, social media, and apps for automated population of user models while addressing system security as well as user privacy. Strategies for enabling the user to set permissions concerning what applications can access their data, how, when, and with whom to share data from a PAL could be explored early to address the privacy and control paradoxes [4].

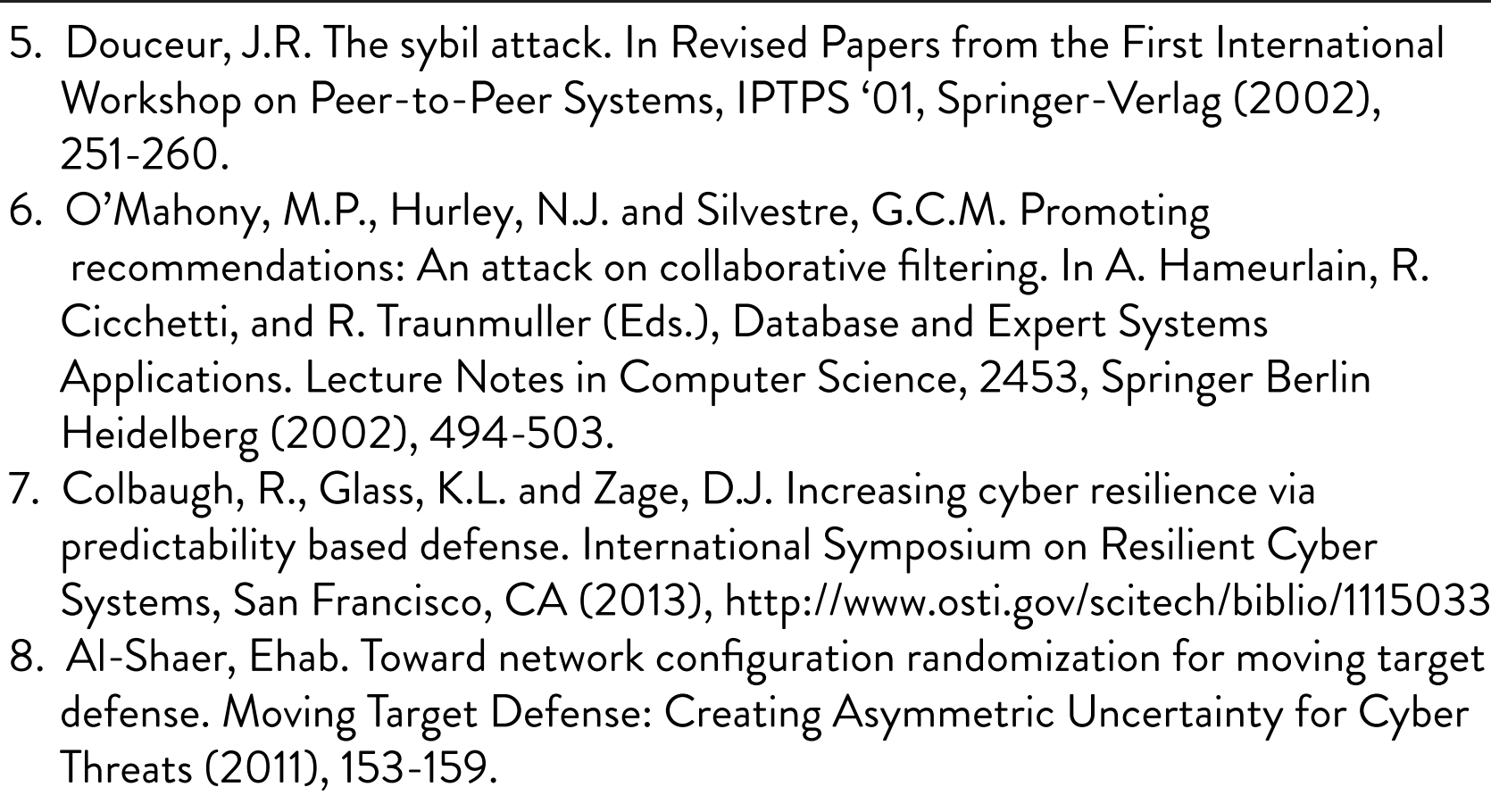

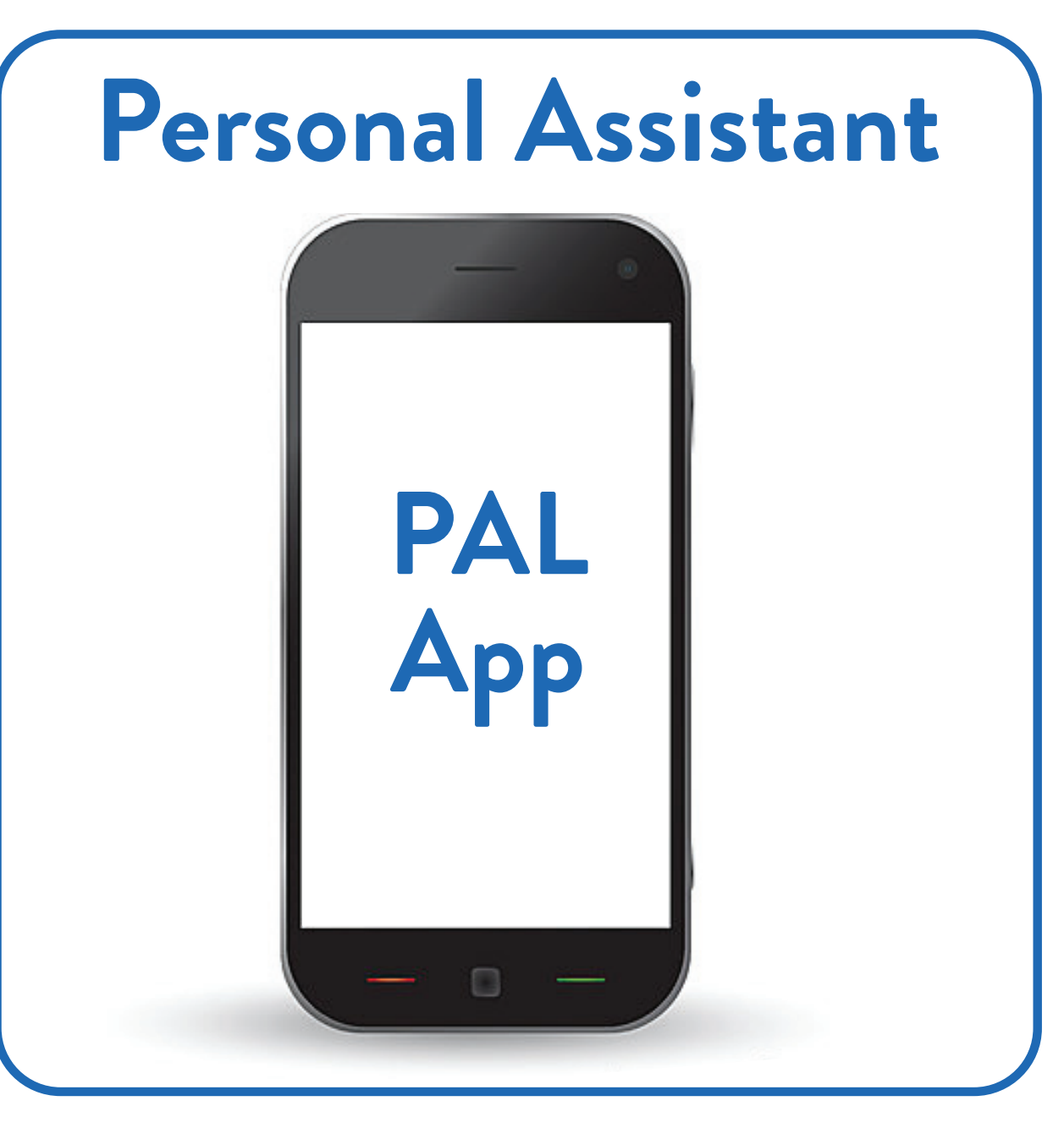

\section{SYSTEMS}

The primary user experience we assume for a PAL is as a mobile app. The architecture would be client-server, where the mobile app acts as a lightweight front-end client to one or more servers providing context awareness and recommendations. The mobile app would be lightweight in the sense that its primary responsibility is sending sensor and user input information to the server and reporting results back from the server to the user through the mobile UI. In some cases, it may cache information locally, but otherwise contains no real actionable data on the device.

Risk: Online training could be modified to include incorrect information, compromising the training that a user would actually receive. By inserting false information into a user's record, recommender models can be altered to provide incorrect information $[5,6]$.

Mitigation: Different algorithms are affected by corrupted data in different ways. Researchers in the Counter Adversarial Data Analytics (CADA) program are working to quantify and classify these different effects [7]

Risk: It is possible to gain some information from network traffic, without needing access to the training content.

Mitigation: A network architecture called Mutable Networks (MUTE) dynamically alters the network topology of a system, making it more difficult for hackers to target particular functions of a network [8]. Dynamic network mutation techniques could be used to obfuscate the activities of a PAL network, making it harder to use network traffic information to gain insight into user activities.

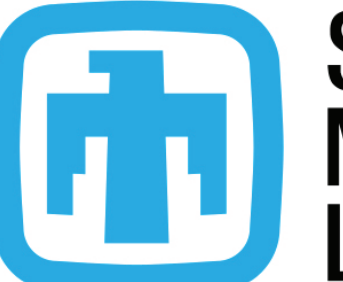

Sandia

National

aboratories

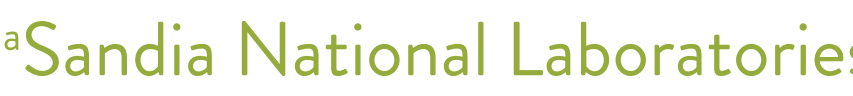

P.O. Box 5800 MS 1325

Albuquerque, NM 87185

\{emraybo, ndfabia, wldavis, rcparks, jtmccl, dtrumbo\}@sandia.gov

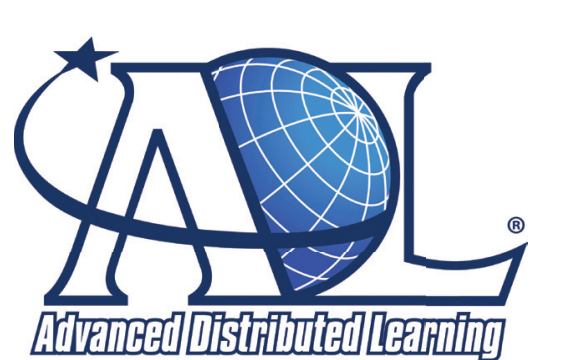

Advanced Distributed Learning Initiative

13501 Ingenuity Drive, Suite 248

Orlando, Florida 32826

\{elaine.raybourn, damon.regan.ctr, paula.durlach\}@adlnet.gov 